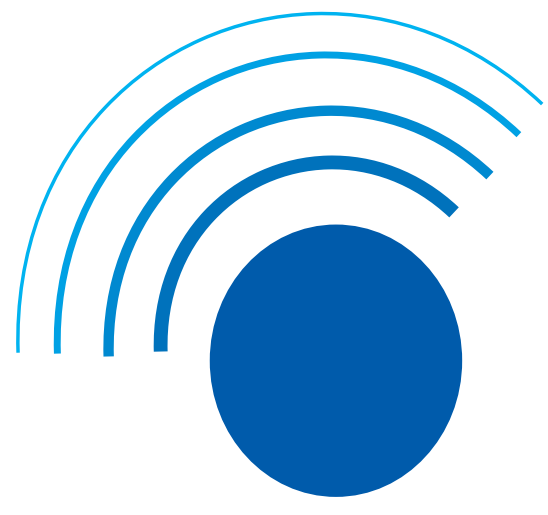

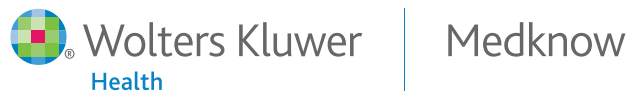
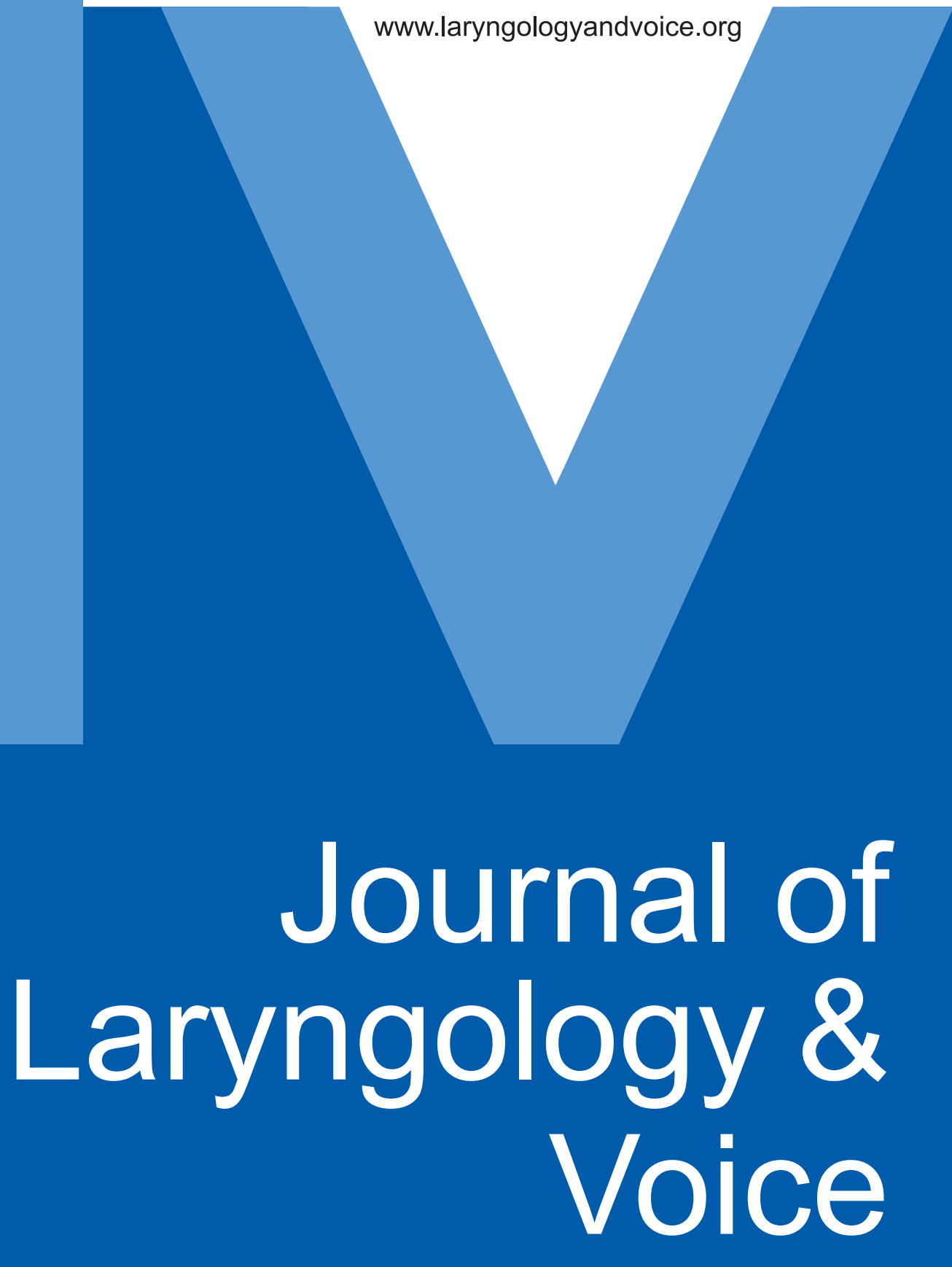

Official Publication of Laryngology and Voice Association 


\section{The effects of}

ILFN-exposure on voice acoustic parameters of commercial cabin crewmembers
Ana Mendes ${ }^{1,2}$, Andreia Graça ${ }^{3}$, Ana Jorge ${ }^{4}$, Mariana Alves-Pereira ${ }^{5}$, Nuno A. A. Castelo Branco ${ }^{6}$, Adriana Freitas $^{1}$, Mara Laranjeira ${ }^{1}$, Íris Bonança ${ }^{1}$

${ }^{1}$ Health School of Polytechnic Institute of Setubal, ${ }^{2}$ Institute of Electronics and Telematics Engineering of Aveiro, ${ }^{3}$ LINADEM - League for the Study and Support for Social Inclusion, ${ }^{4}$ Institute of Accounting and Administration of Lisbon, ${ }^{5} \mathrm{CIEG}$ - Faculty of Economics and Management \& ERISA School of Health Sciences, Lusófona University, Lisbon, ${ }^{6}$ Human Performance Center, Alverca, Portugal

\begin{abstract}
Background: Long-term exposure to infrasound and low frequency noise (ILFN $<500 \mathrm{~Hz}$, including infrasound) can lead to the development of vibroacoustic disease (VAD). VAD is a systemic pathology characterized by the abnormal growth of extracellular matrices in the absence of inflammatory processes, namely of collagen and elastin, both of which are abundant in the basement membrane zone of the vocal folds. ILFN-exposed workers include pilots, cabin crewmembers, restaurant workers, ship machinists and, in previous studies, even though they did not present vocal symptoms, ILFN-exposed workers had significant different voice acoustic patterns (perturbation and temporal measures) when compared with normative population. Study Aims: The present study investigates the effects of age and years of occupational ILFN-exposure on voice acoustic parameters of 37 cabin crewmembers: 12 males and 25 females. Specifically, the goals of this study are to: 1) Verify if acoustic parameters change over the age and years of ILFN-exposure and 2) Determine if there is any interaction between age and years of ILFNexposure on voice acoustic parameters of crewmembers. Materials and Methods: Spoken phonatory tasks were recorded with a C420"I PP AKG head-worn microphone and a DA-P1 Tascam DAT. Acoustic analyses were performed using KayPENTAX Computer Speech Lab and Multi-Dimensional Voice Program. Acoustic parameters included speaking fundamental frequency, perturbation measures (jitter, shimmer and harmonicto-noise ratio), temporal measures (maximum phonation time and s/z ratio) and voice tremor frequency. Results: One-way ANOVA analysis revealed that as the number of ILFN-exposure years increased male cabin crewmembers presented significant different shimmer values of $/ \mathrm{i} /$ as well as tremor frequency of $/ \mathrm{u} /$. Females presented significantly different jitter $\%$ of $/ \mathrm{i}$, a, o/ $(p<0.05)$. Lastly, Two-way ANOVA analysis revealed that for females, there was a significant interaction between age and occupational ILFN-exposure for voice acoustic parameters, namely for jitter's mean for /a, o/ and shimmer's (\%) mean for /a, i/ $(p<0.05)$. Discussion and Conclusion: These perturbation measure patterns may be indicative of histological changes within the vocal folds as a result of ILFN-exposure. The results of this study suggest that voice acoustic analysis may be an important tool for confirming ILFN-induced health effects.
\end{abstract}

Key words: Infrasound and low frequency noise-exposure, vibroacoustic disease, vocal folds, voice acoustic analysis

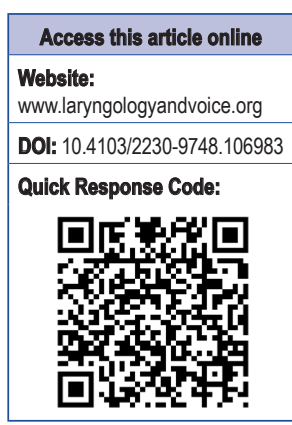

\section{INTRODUCTION}

Vibroacoustic disease (VAD) is a systemic pathology caused by long-term exposure to infrasound and low frequency noise (ILFN), specifically frequencies below $500 \mathrm{~Hz}$, including

Address for correspondence:

Dr. Íris Bonança, Polytechnic Institute of Setubal, School of Health Campus do IPS, Estefanilha, 2914-503 Setúbal, Portugal.

E-mail: iris.bonanca@ess.ips.pt infrasound. ${ }^{[1-3]}$ This acoustical phenomenon affects organs and systems other than hearing, particularly the respiratory tract. The hallmark of VAD is the abnormal growth of extracellular matrices, namely collagen and elastin, in the absence of inflammatory processes.

The effects of VAD and ILFN exposure have been described in several human and animal organs and systems, other than hearing, such as: Cardiac system (thickening of 
cardiac structures seen through echocardiography, ${ }^{[3,4]}$ pericardial thickening, as seen through light and electron microscopy), ${ }^{[1,3]}$ neurological system (cerebral lesions, increased latency of nerve conduction, primitive reflexes, facial dyskinesia, epilepsy), ${ }^{[5,6]}$ intestinal system (duodenal alterations in rats which may be equivalent to the ulcers and dyspeptic symptoms reported in human VAD patients, superficial erosions and widespread cell death with microvilli coalescence and fusion), ${ }^{[7]}$ psychological system (depression, increased irritability and aggressiveness, a tendency for isolation, and decreased cognitive skills)..$^{[1,2]}$ Furthermore, potential genotoxic effects, inducing an increased frequency of sister chromatid exchanges, ${ }^{[8]}$ immunological changes, ${ }^{\left[{ }^{[0]}\right.}$ and digestive symptoms (i.e., cramps, nauseas, diarrhea) with gastrointestinal lesions have been described. ${ }^{[7]}$

VAD affects particularly the respiratory tract as is reflected in thickening of respiratory system as seen through electron microscopy in biopsy material and autopsy findings. ${ }^{[10-13]}$ ILFNexposed animal models also revealed thickening of respiratory tract structures due to the abnormal growth of collagen. ${ }^{[14]}$

Non-productive cough, hoarseness, repeated upper and lower respiratory infections, bronchitis (in smokers and nonsmokers alike) and respiratory insufficiency were included in respiratory complaints of older ILFN-exposed workers and VAD patients. ${ }^{[2,15,16]}$ Lung fibrosis was also diagnosed in a group of ILFN-exposed workers, both with and without respiratory complaints..$^{[15,16]}$

Bronchoscopic evaluation of 30 airline crewmembers (10 males and 20 females) revealed pink patches of the trachea and vocal folds as well as bleeding events limited to the lower portion of the epithelium, i.e., closer to the vocal folds' basal lamina. ${ }^{[11,12]}$

Histologically, the vocal folds' stratified squamous epithelium and the lamina propria's superficial layer are held together by the basement membrane zone (BMZ), which is susceptible to injury caused by the impact of vibration and shearing forces. To strengthen this vibratory system, the BMZ is composed of three layers: The plasma membrane, the lamina densa and the sub-basement membrane area. Basal cells composed of proteins extend from the plasma membrane to the lamina densa, which is composed by Type IV collagen fibers. From the lamina densa to the sub-basement membrane area, looping fibers are wrapped by Type III collagen fibers. These fragile connecting links can be damaged by injury, nodules or other benign tumors. ${ }^{\mid 17,18]}$

Given the collagen content of the vocal folds and the lesions observed in the bronchoscopic studies (i.e., pink patches on the basal lamina) a question arises: Could the laryngeal system be itself a target for ILFN?

Voice acoustic analysis is a sensitive instrument that can be used to trace laryngeal and respiratory changes. ${ }^{[19,20]}$ Three studies compared voice acoustic measures of normative data of voice acoustic parameters of VAD patients $(N=9)$ and ILFNexposure workers (36 commercial airline pilots and 37 airline cabin crewmembers). ${ }^{[21-23]}$ Results revealed that the three groups presented significant differences of perturbation and temporal measures when compared with the norms $(p<0.05)$. Jitter values were increased and shimmer values were decreased. Maximum phonation time and $\mathrm{s} / \mathrm{z}$ ration were decreased. These results might reflect slight differences of mass, tension and biomechanical characteristics of vocal folds as well as an inefficient coordination between the respiratory and phonatory system. ${ }^{[20]}$ These patterns might indicate small morphological and physiological changes of the respiratory and laryngeal systems which had been reported in previous animal models and human studies. Fundamental frequency, harmonic-to-noise ratio and frequency tremor were within normal limits. However the question, about these significant findings, might be related with age and/or number of years of occupational ILFN-exposure.

The objective of the present study was to investigate the effects of age and years of occupational ILFN-exposure on voice acoustic parameters of 37 cabin crewmembers, with the following specific goals: 1) determine if there are significant differences in vocal acoustic parameters among four occupational ILFN-exposure intervals ((i.e., 4-38 years), and 2) determine if there are significant differences of vocal acoustic parameters between the four occupational ILFNexposure and three age intervals (i.e., 29-60 years).

\section{MATERIALS AND METHODS}

\section{Subjects}

Thirty-seven airline cabin crewmembers voluntarily participated in this study: 12 males (mean age of 43 years, range 29-60 years) and 25 females (mean age of 45 years, range 26-62 years). All were ILFN-exposed workers, classified as Type II - Professional voice users, whose voice is an integral part of their profession and whose work performance can be compromised by a moderate voice problem. ${ }^{[24]}$ All subjects presented hearing abilities and middle ear functions within normal limits.

The male group presented an occupational ILFN-exposure mean of 19 years, ranging from 7 to 36 years. Five $(5 / 12=42 \%)$ were smokers and nine had mild alcoholic habits (1-2 glasses day/several years). Two men reported allergy-related 
respiratory complaints. Five males reported voice complaints and two of them were diagnosed with laryngeal papilloma and vocal polyps.

The female group presented an occupational ILFN-exposure mean of 22 years, ranging from 4 to 38 years. Twelve $(12 / 25=48 \%)$ were smokers and seven had mild alcoholic habits (1-2 glasses day/ several years) [Tables 1-3]. Ten reported respiratory complaints and nine reported complaints of hoarseness.

It should be noted that this study does not include a control group due to three main reasons: 1) Previous studies already reported that voice acoustic parameters of ILFN-exposure group are different from normative data; ${ }^{[21-23]}$ 2) this study aims to assess the effects of ILFN-exposure on voice acoustic parameters and 3) this is a correlational study between two different variables (i.e., age and occupational ILFN-exposure). Therefore, it is not required to analyze the normative behavior because it does not interfere with the final results or the conclusion. Moreover, nowadays it is very difficult to recruit subject that were never exposed to ILFN generators (i.e., trains, subway, airplanes, automobiles). Even those that live out of the big cities had same kind of ILFN-exposure (i.e., windmills, tractors).

\section{Phonatory tasks}

Subjects performed speaking tasks in the standing position. Before recordings, subjects briefly warmed-up their voice by performing phonational and dynamic ranges as well as reading aloud the European Portuguese phonetically balanced text called "O Sol", ${ }^{[25]}$ (The Sun). Subjects were asked to sustain /a, i, o, u/ three times each for 6 seconds. Even though 6 to 10 trials are recommended for perturbations measures ${ }^{\mid 26]}$ only three trials of each task were performed to avoid subjects' fatigue. Subjects also sustained /a, s, z/ three times each for as long as they could.

\begin{tabular}{|c|c|c|c|c|c|c|c|c|}
\hline S.N. & ID & Gender & $\begin{array}{l}\text { Age } \\
\text { (yrs) }\end{array}$ & $\begin{array}{l}\text { Occupational } \\
\text { ILFN-exposure } \\
\text { (yrs) }\end{array}$ & $\begin{array}{c}\text { Smoker } \\
(\mathrm{Y} / \mathrm{N})\end{array}$ & $\begin{array}{c}\text { Alcoholic } \\
\text { habits } \\
(\mathrm{Y} / \mathrm{N})\end{array}$ & $\begin{array}{c}\text { Respiratory } \\
\text { complaints } \\
\text { (Y/N) }\end{array}$ & $\begin{array}{c}\text { Vocal } \\
\text { complaints } \\
(\mathrm{Y} / \mathrm{N})\end{array}$ \\
\hline 1 & $J R$ & $M$ & 60 & 35 & $Y$ & $Y$ & $\mathrm{Y}$ & $\mathrm{N}$ \\
\hline 2 & $\mathrm{CV}$ & $M$ & 57 & 27 & $\mathrm{~N}$ & Y & $\mathrm{N}$ & Y \\
\hline 3 & CF & M & 59 & 34 & $\mathrm{~N}$ & $Y$ & $\mathrm{~N}$ & $Y$ \\
\hline 4 & RG & $\mathrm{M}$ & 32 & 10 & $\mathrm{~N}$ & $Y$ & $Y$ & $Y$ \\
\hline 5 & PB & $M$ & 29 & 8 & Y & $Y$ & $\mathrm{~N}$ & $\mathrm{~N}$ \\
\hline 6 & $\mathrm{JB} 1$ & M & 31 & 7 & $Y$ & Y & $\mathrm{N}$ & $\mathrm{N}$ \\
\hline 7 & MA1 & $\mathrm{M}$ & 44 & 20 & $\mathrm{~N}$ & $Y$ & $\mathrm{~N}$ & $\mathrm{~N}$ \\
\hline 8 & LM2 & M & 60 & 33 & $Y$ & $Y$ & $\mathrm{~N}$ & $\mathrm{~N}$ \\
\hline 9 & NM & M & 35 & 10 & $Y$ & $\mathrm{~N}$ & $\mathrm{~N}$ & $\mathrm{~N}$ \\
\hline 10 & $\mathrm{HM}$ & $M$ & 31 & 10 & $\mathrm{~N}$ & $\mathrm{~N}$ & $\mathrm{~N}$ & Y \\
\hline 11 & $\mathrm{JD}$ & $\mathrm{M}$ & 34 & 14 & $\mathrm{~N}$ & $\mathrm{~N}$ & $\mathrm{~N}$ & $\mathrm{~N}$ \\
\hline 12 & $\mathrm{JB} 2$ & $M$ & 43 & 20 & $Y$ & $Y$ & $\mathrm{~N}$ & Y \\
\hline 13 & JS & $\mathrm{F}$ & 31 & 7 & $Y$ & $\mathrm{~N}$ & Y & $\mathrm{N}$ \\
\hline 14 & AA & $\mathrm{F}$ & 57 & 34 & $\mathrm{~N}$ & $\mathrm{~N}$ & $\mathrm{~N}$ & $\mathrm{~N}$ \\
\hline 15 & $S L$ & $\mathrm{~F}$ & 57 & 35 & $Y$ & $Y$ & $Y$ & $\mathrm{~N}$ \\
\hline 16 & IP & $\mathrm{F}$ & 32 & 10 & Y & $\mathrm{N}$ & $\mathrm{N}$ & Y \\
\hline 17 & $\mathrm{AF} 1$ & $\mathrm{~F}$ & 37 & 15 & $\mathrm{~N}$ & $\mathrm{~N}$ & $Y$ & Y \\
\hline 18 & $\mathrm{ME}$ & $\mathrm{F}$ & 53 & 35 & $\mathrm{~N}$ & $\mathrm{~N}$ & $\mathrm{~N}$ & Y \\
\hline 19 & MO1 & $\mathrm{F}$ & 51 & 30 & $\mathrm{~N}$ & $Y$ & $\mathrm{~N}$ & $\mathrm{~N}$ \\
\hline 20 & LM1 & $\mathrm{F}$ & 26 & 4 & $\mathrm{~N}$ & $\mathrm{~N}$ & $\mathrm{~N}$ & $\mathrm{~N}$ \\
\hline 21 & $\mathrm{MP}$ & $\mathrm{F}$ & 32 & 10 & $Y$ & $Y$ & $\mathrm{~N}$ & $\mathrm{~N}$ \\
\hline 22 & MB & $\mathrm{F}$ & 40 & 18 & $Y$ & $\mathrm{~N}$ & $\mathrm{~N}$ & $\mathrm{~N}$ \\
\hline 23 & MO2 & $\mathrm{F}$ & 44 & 20 & $\mathrm{~N}$ & $\mathrm{~N}$ & $\mathrm{~N}$ & $\mathrm{~N}$ \\
\hline 24 & SB & $\mathrm{F}$ & 41 & 20 & $\mathrm{~N}$ & $\mathrm{~N}$ & $\mathrm{~N}$ & $Y$ \\
\hline 25 & $\mathrm{CM}$ & $\mathrm{F}$ & 35 & 13 & $Y$ & $\mathrm{~N}$ & $\mathrm{~N}$ & $Y$ \\
\hline 26 & $M C$ & $\mathrm{~F}$ & 59 & 18 & $\mathrm{~N}$ & $\mathrm{~N}$ & $Y$ & $\mathrm{~N}$ \\
\hline 27 & MA3 & $\mathrm{F}$ & 56 & 35 & $Y$ & $\mathrm{~N}$ & $\mathrm{~N}$ & $\mathrm{~N}$ \\
\hline 28 & FC & $\mathrm{F}$ & 60 & 38 & $Y$ & $Y$ & $\mathrm{~N}$ & $\mathrm{~N}$ \\
\hline 29 & $\mathrm{CC} 2$ & $\mathrm{~F}$ & 39 & 16 & $Y$ & $Y$ & $Y$ & $\mathrm{~N}$ \\
\hline 30 & AS & $\mathrm{F}$ & 62 & 31 & $\mathrm{~N}$ & $\mathrm{~N}$ & $\mathrm{~N}$ & $\mathrm{~N}$ \\
\hline 31 & $\mathrm{DF}$ & $\mathrm{F}$ & 58 & 38 & $Y$ & $Y$ & $Y$ & $Y$ \\
\hline 32 & MA4 & $\mathrm{F}$ & 58 & 32 & $\mathrm{~N}$ & $\mathrm{~N}$ & $Y$ & $\mathrm{~N}$ \\
\hline 33 & MA5 & $\mathrm{F}$ & 41 & 18 & $\mathrm{~N}$ & $\mathrm{~N}$ & $\mathrm{~N}$ & $Y$ \\
\hline 34 & $\mid A$ & $\mathrm{~F}$ & 36 & 17 & $Y$ & $\mathrm{~N}$ & $Y$ & $\mathrm{~N}$ \\
\hline 35 & VM & $\mathrm{F}$ & 35 & 17 & $\mathrm{~N}$ & $\mathrm{~N}$ & $\mathrm{~N}$ & $\mathrm{~N}$ \\
\hline 36 & $A G$ & $\mathrm{~F}$ & 32 & 10 & $Y$ & $\mathrm{~N}$ & $Y$ & $Y$ \\
\hline 37 & $A B$ & $\mathrm{~F}$ & 55 & 25 & $\mathrm{~N}$ & $Y$ & $Y$ & $Y$ \\
\hline
\end{tabular}




\section{Equipment}

Phonatory tasks were recorded in a quiet environment $(>50 \mathrm{~dB})$ at the Sindicato Nacional do Pessoal de Voo da Aviação Civil, in Lisbon. Voice productions were captured with an AKG C420 III PP high-quality cardioid type headset microphone placed $3 \mathrm{~cm}$ from the right corner of the mouth and at a $45^{\circ}$ angle. ${ }^{|27|}$ The acoustic signal was amplified with a phantom RANE MS-16. An Agilente 8494A attenuator was activated for loud phonations to avoid peak clipping. Voice samples were recorded to a portable Tascam DA-P1 Digital Audiotape Recorder (DAT). For calibration purposes, a $500 \mathrm{~Hz}$ tone of $80 \mathrm{~dB} \mathrm{SPL}$, at $2 \mathrm{~cm}$ distance from sound source to microphone, was recorded onto each digital audiotape. Prior to measuring each subject's productions, the calibration tone was digitized and served as a reference tone calculated by KayPENTAX Computer Speech Lab (CSL), model 4500. ${ }^{[28]}$

For the acoustic analyses, a HP dx5150 with the CSL external sound card was used with the Multi-Dimensional Voice Program (MDVP) software, model 5105. All samples were amplified with the Audacity software, model 1.2.6. and digitized at a rate of $25.0 \mathrm{kHz} .^{[29]}$

\section{Acoustic analysis}

Acoustic measurements of the sustained vowels were fundamental frequency $\left(\mathrm{F}_{0} ; \mathrm{Hz}\right)$, jitter $(\%)$, shimmer $(\%)$, harmonic-to-noise ratio (HNR) and voice tremor $\left(\mathrm{F}_{\mathrm{ftr}} ; \mathrm{Hz}\right)$. Maximum phonation duration (MPD; s) and s/z ratio were obtained from sustained /a, s, z/, respectively.

\section{Statistical analysis}

This transversal study looked at the effects of occupational ILFN-exposure of four intervals (i.e., <9, [9-20], [20-26], >26) and three age intervals (i.e., $<37,[37 ; 45],>45$ ) on voice acoustic parameters of 37 cabin crewmembers [Table 4]. It was designed to measure and compare the similarities and differences of the voice acoustic parameters among these four intervals. ${ }^{[30]}$

Descriptive statistics consisted of mean and standard deviation analysis of all numerical variables. It was performed with Microsoft Office Excel 2003.

Inferential statistics were performed with Statistical Package for Social Sciences (SPSS) PASW Statistics18 consisted on One and Two-way ANOVA and statistical significance was established at $\alpha=0.05$ for each variable. Vocal acoustic parameters were treated separately for each gender, due to significant differences found in all spoken acoustic parameters, as expected. One-way ANOVA was performed to detect significant differences of voice acoustic parameters between both genders as well as among four occupational

\begin{tabular}{|c|c|c|c|c|c|}
\hline \multirow[t]{2}{*}{ Gender } & \multirow[t]{2}{*}{$\mathrm{N}$} & \multicolumn{2}{|c|}{ Age } & \multicolumn{2}{|c|}{$\begin{array}{l}\text { Occupational } \\
\text { ILFN-exposure }\end{array}$} \\
\hline & & Mean & Range & Mean & Range \\
\hline M & 12 & 43 & $29-60$ & 19 & 36 \\
\hline $\mathrm{F}$ & 25 & 45 & $26-62$ & 22 & 38 \\
\hline Total & 37 & & & & \\
\hline
\end{tabular}

\begin{tabular}{|c|c|c|c|c|}
\hline \multirow[t]{2}{*}{ S.N. } & \multirow{2}{*}{$\begin{array}{l}\text { Occupational } \\
\text { ILFN-exposure } \\
\text { (years) }\end{array}$} & \multicolumn{3}{|c|}{$\mathbf{N}$} \\
\hline & & M & $F$ & Total \\
\hline 1 & $<9$ & 2 & 2 & 4 \\
\hline 2 & {$[9 ; 20]$} & 4 & 11 & 15 \\
\hline 3 & {$[20 ; 26]$} & 2 & 3 & 5 \\
\hline \multirow[t]{2}{*}{4} & $\geq 26$ & 4 & 9 & 13 \\
\hline & Total & 12 & 25 & 37 \\
\hline
\end{tabular}

Table 4: Subjects' distribution of age and occupational ILFN-exposure (years)

\begin{tabular}{lccc} 
Age & $\begin{array}{c}\text { Professional activity } \\
\text { intervals (years) }\end{array}$ & \multicolumn{2}{c}{$\mathbf{N}$} \\
\cline { 3 - 4 } & $<9$ & $\mathbf{M}$ & $\mathbf{F}$ \\
\hline$<37$ & {$[9 ; 20]$} & 4 & 2 \\
& {$[9 ; 20]$} & - & 6 \\
{$[37 ; 45]$} & {$[20 ; 26]$} & 2 & 2 \\
& {$[9 ; 20]$} & - & 1 \\
$>45$ & {$[20 ; 26]$} & - & 1 \\
& $\geq 26$ & 4 & 9 \\
Total & & 12 & 25 \\
\hline
\end{tabular}

ILFN-exposure intervals. Two-way ANOVA was performed to detect significant differences between occupational ILFNexposure intervals and age intervals.

\section{RESULTS}

The mean $\mathrm{F}_{0}$ for both genders in all vowels increased as the occupational ILFN-exposure intervals (years) increased. This pattern was more evident for the male group [Table 5].

The mean of jitter (\%) changed throughout the four professional activity intervals for both genders and for all vowels. For the female group, One-way ANOVA revealed significant differences of jitter for the /a, i, o/ among the four occupational ILFN-exposure intervals $(p<0.05)$ [Table 6]. On the third occupational ILFN-exposure interval (i.e., [20-26] years), females presented a jitter (\%) increment for the three vowels [Figures 1-3].

The mean shimmer $(\mathrm{dB} ; \%)$ changed throughout the four occupational ILFN-exposure intervals for both genders overall. 


\begin{tabular}{|c|c|c|c|c|c|}
\hline \multirow[t]{3}{*}{ Vowels } & \multicolumn{5}{|c|}{ Males $\mathrm{F}_{0}(\mathrm{~Hz})$} \\
\hline & \multicolumn{5}{|c|}{ Mean \pm SD } \\
\hline & $<9$ & {$[9 ; 20]$} & {$[20 ; 26]$} & $\geq 26$ & $p$ \\
\hline$/ \mathrm{a} /$ & $111.33 \pm 1.32$ & $112.83 \pm 15.27$ & $116.66 \pm 43.08$ & $162.88 \pm 40.29$ & 0.148 \\
\hline /i/ & $124.98 \pm 22.37$ & $114.82 \pm 17.18$ & $131.75 \pm 64.19$ & $193.95 \pm 59.97$ & 0.148 \\
\hline$/ 0 /$ & $117.93 \pm 1.00$ & $117.72 \pm 13.58$ & $124.93 \pm 18.76$ & $158.48 \pm 31.83$ & 0.115 \\
\hline \multirow[t]{3}{*}{$/ \mathrm{u} /$} & $127.82 \pm 20.19$ & $126.16 \pm 12.08$ & $134.12 \pm 0.75$ & $180.69 \pm 45.18$ & 0.108 \\
\hline & \multicolumn{5}{|c|}{ Females $F_{0}(H z)$} \\
\hline & \multicolumn{5}{|c|}{ Mean \pm SD } \\
\hline$/ \mathrm{a} /$ & $207,40 \pm 10,63$ & $194,92 \pm 37,03$ & $203,37 \pm 34,15$ & $213,90 \pm 67,98$ & 0.868 \\
\hline /i/ & $211,81 \pm 14,38$ & $205,65 \pm 31,25$ & $223,53 \pm 25,12$ & $229,56 \pm 72,65$ & 0.758 \\
\hline$/ 0 /$ & $202,97 \pm 9,52$ & $193,18 \pm 32,30$ & $201,65 \pm 41,40$ & $208,10 \pm 61,13$ & 0.909 \\
\hline$/ \mathrm{u} /$ & $222,02 \pm 1,58$ & $203,46 \pm 31,23$ & $227,53 \pm 30,08$ & $222,67 \pm 72,12$ & 0.799 \\
\hline
\end{tabular}

\begin{tabular}{|c|c|c|c|c|c|}
\hline \multirow[t]{3}{*}{ Vowels } & \multicolumn{5}{|c|}{ Males Jitter (\%) } \\
\hline & \multicolumn{5}{|c|}{ Mean \pm SD } \\
\hline & $<9$ & {$[9 ; 20]$} & {$[20 ; 26]$} & $\geq 26$ & $p$ \\
\hline$/ \mathrm{a} /$ & $1.18 \pm 0.92$ & $0.89 \pm 0.71$ & $0.47 \pm 0.02$ & $0.39 \pm 0.11$ & 0.360 \\
\hline /i/ & $0.64 \pm 0.18$ & $0.38 \pm 0.11$ & $0.51 \pm 0.17$ & $0.73 \pm 0.66$ & 0.695 \\
\hline$/ 0 /$ & $1.14 \pm 1.24$ & $0.45 \pm 0.23$ & $0.62 \pm 0.44$ & $0.36 \pm 0.14$ & 0.370 \\
\hline \multirow[t]{3}{*}{$/ \mathrm{u} /$} & $0.82 \pm 0.54$ & $0.77 \pm 0.31$ & $0.59 \pm 0.01$ & $0.94 \pm 0.47$ & 0.772 \\
\hline & \multicolumn{5}{|c|}{ Females Jitter (\%) } \\
\hline & \multicolumn{5}{|c|}{ Mean \pm SD } \\
\hline$/ \mathrm{a} /$ & $1.22 \pm 0.28$ & $0.58 \pm 0.29$ & $1.34 \pm 1.23$ & $0.54 \pm 0.24$ & $0.036^{*}$ \\
\hline /i/ & $0.72 \pm 0.25$ & $0.60 \pm 0.37$ & $1.84 \pm 0.96$ & $0.90 \pm 0.51$ & 0.010 * \\
\hline$/ 0 /$ & $0.94 \pm 0.45$ & $0.62 \pm 0.26$ & $1.42 \pm 0.90$ & $0.57 \pm 0.25$ & $0.015^{*}$ \\
\hline$/ \mathrm{u} /$ & $1.72 \pm 0.49$ & $0.89 \pm 0.28$ & $1.20 \pm 0.19$ & $2.24 \pm 3.67$ & 0.624 \\
\hline
\end{tabular}

${ }^{*} p<0.05$

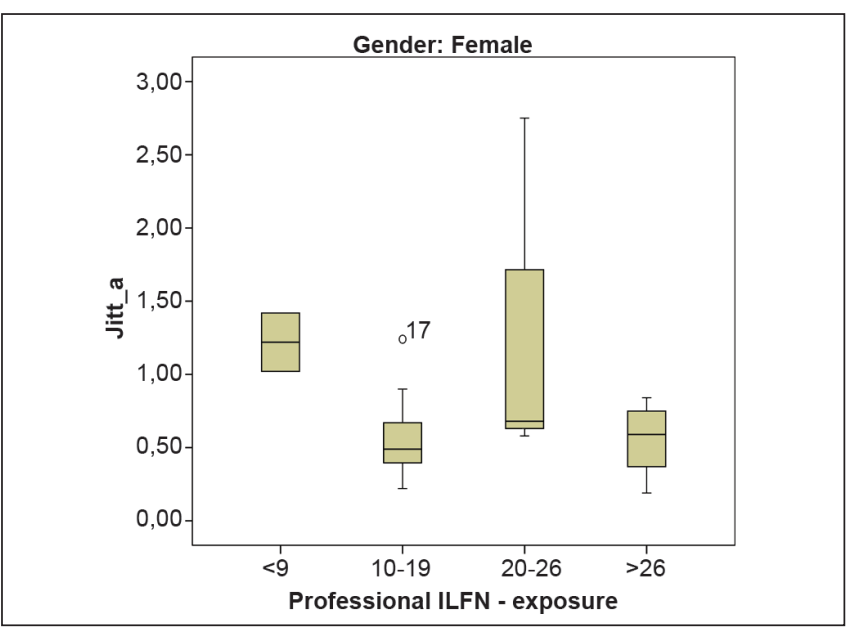

Figure 1: Box plot of jitter (\%) of females for /a/. This variable changed throughout the four occupational ILFN-exposure intervals. On the third professional activity interval (i.e., 20-26 years), jitter presented an increment and a larger dispersion among the maximum values

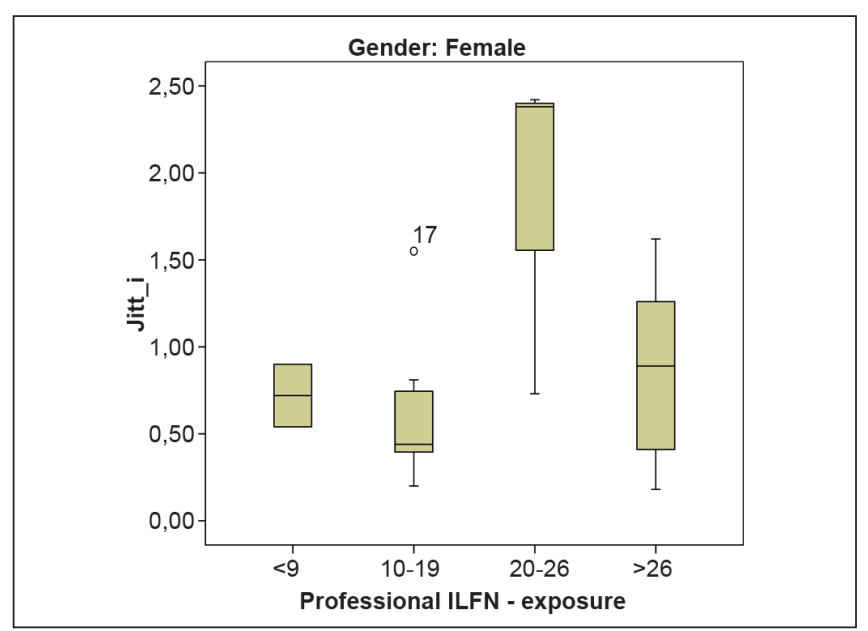

Figure 2: Box plot of jitter (\%) of females for /i/. This variable changed throughout the four occupational ILFN-exposure intervals. On the third professional activity interval (i.e., 20-26 years), jitter presented an increment and a larger dispersion among the minimum values 
There was a tendency for the mean value of shimmer to increase throughout the first three occupational ILFN-exposure intervals and to decrease on the fourth interval. For the male group, One-way ANOVA revealed a significant difference of shimmer for $/ \mathrm{i}$ / among the four occupational ILFN-exposure intervals $(p=0.012)$ [Table 7]. On the third occupational ILFN-exposure interval (i.e. 20-26 years), there was a significant increment $(p=0.012)$.

HNR's mean was steady throughout the four occupational ILFN-exposure intervals for both genders [Table 8].

Mean values for frequency tremor $\left(\mathrm{F}_{\mathrm{ftr}}\right)$ of male and female subjects for the /a, i, o, u/ were presented in Table 9. Fftr's mean had a tendency to increase during the four occupational ILFN-exposure intervals for both genders. Male subjects presented a significant increment of $\mathrm{F}_{\mathrm{ftr}}$ for the $/ \mathrm{u} /(p=0.035)$.

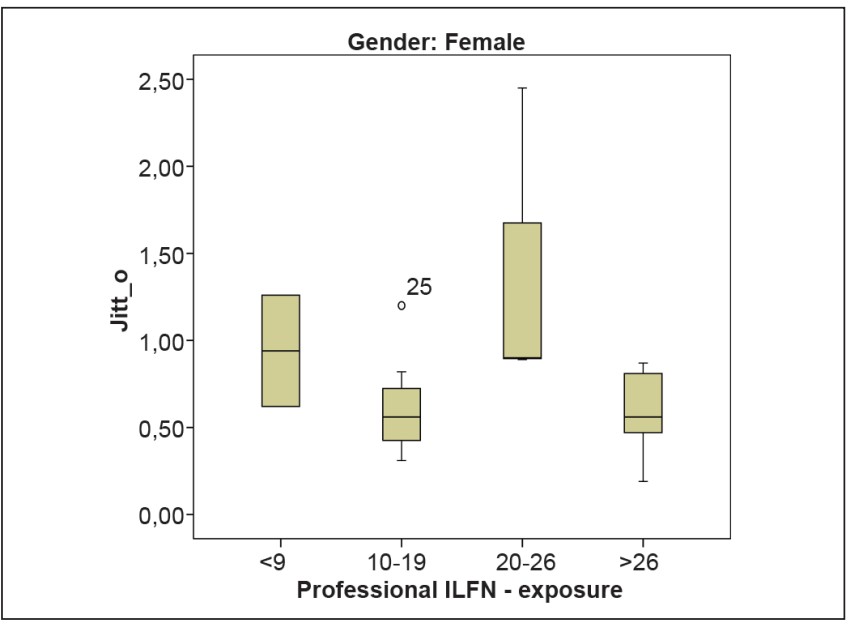

Figure 3: Box plot of jitter (\%) of females for $/ \mathrm{O} /$. This variable changed throughout the four occupational ILFN-exposure intervals. On the third professional activity interval (i.e., 20-26 years), jitter presented an increment and a larger dispersion among the maximum values
Temporal measures, MPD and s/z were steady throughout the four occupational ILFN-exposure intervals for both genders [Table 10].

Two-way ANOVA was performed to analyse the interaction of age (years) and occupational ILFN-exposure intervals (years) only for female group. There was insufficient data to analyse the male group $(N=12)$. A significant interaction existed for the variables jitter and shimmer $(\%)$ for /a, o/ and /a, i/ respectively $(p<0.05)$ [Table 11]. There was no interaction for $\mathrm{F}_{0}, \mathrm{HNR}$ and $\mathrm{F}_{\mathrm{ftr}}$ for all vowels [Table 12] and temporal measures [Table 13].

\section{DIsCUSSION}

The purpose of the present study was to analyse the effects of occupational ILFN-exposure (i.e., 4-38 years in four intervals: $<9,[9 ; 20],[20 ; 26], \geq 26)$ in voice acoustic parameters of 37 cabin crewmembers (12 males and 25 females). Secondly, the interaction between age (i.e., 29-60 years in three intervals: $<37$, [37;45], >45) and occupational ILFN-exposure was analysed on the same acoustic parameters.

The three previous studies that compared normative voice acoustic data with those obtained with VAD patients and ILFN exposed groups (pilots and crewmembers) revealed significant differences in perturbation measures, specifically higher jitter and lower shimmer. ${ }^{[21-23]}$ These studies raised the question whether these differences were related to occupational ILFN-exposure. The present study revealed that the mean speaking $\mathrm{F}_{0}$ of all vowels increased with increasing occupational ILFN-exposure. This pattern was more marked in males [Table 5]. The jitter (\%) in females changed significantly $(p<0.05)$ throughout the four occupational ILFN-exposure intervals. A pattern of decreasing, increasing and decreasing again on the last interval of ILFN-exposure is evident. The

Table 7: Mean, standard deviation and One-way ANOVA of shimmer for male and female airline crew members

\begin{tabular}{|c|c|c|c|c|c|}
\hline \multirow[t]{3}{*}{ Vowels } & \multicolumn{5}{|c|}{ Males Shimmer (\%) } \\
\hline & \multicolumn{5}{|c|}{ Mean \pm SD } \\
\hline & $<9$ & {$[9 ; 20]$} & {$[20 ; 26]$} & $\geq 26$ & $p$ \\
\hline$/ \mathrm{a} /$ & $1.20 \pm 0.29$ & $2.99 \pm 1.65$ & $2.75 \pm 1.57$ & $1.34 \pm 1.05$ & 0.372 \\
\hline /i/ & $1.83 \pm 0.46$ & $1.59 \pm 0.37$ & $2.47 \pm 0.15$ & $1.11 \pm 0.40$ & $0.012 *$ \\
\hline$/ 0 /$ & $0.94 \pm 0.11$ & $2.20 \pm 1.38$ & $4.25 \pm 0.42$ & $1.58 \pm 0.87$ & 0.113 \\
\hline \multirow[t]{3}{*}{$/ \mathrm{u} /$} & $2.06 \pm 1.16$ & $2.29 \pm 1.31$ & $1.78 \pm 1.28$ & $1.31 \pm 0.55$ & 0.408 \\
\hline & \multicolumn{5}{|c|}{ Females Shimmer (\%) } \\
\hline & \multicolumn{5}{|c|}{ Mean \pm SD } \\
\hline$/ \mathrm{a} /$ & $2.79 \pm 0.13$ & $2.32 \pm 1.07$ & $3.61 \pm 1.73$ & $2.04 \pm 0.71$ & 0.157 \\
\hline /i/ & $1.54 \pm 0.45$ & $2.04 \pm 1.41$ & $2.34 \pm 1.76$ & $1.56 \pm 1.00$ & 0.747 \\
\hline $\mid 0 /$ & $2.74 \pm 1.31$ & $3.06 \pm 4.44$ & $3.44 \pm 1.16$ & $1.62 \pm 0.76$ & 0.724 \\
\hline$/ \mathrm{u} /$ & $4.43 \pm 3.78$ & $2.72 \pm 2.05$ & $3.96 \pm 1.17$ & $5.21 \pm 8.64$ & 0.799 \\
\hline
\end{tabular}




\begin{tabular}{|c|c|c|c|c|c|}
\hline \multirow[t]{3}{*}{ Vowels } & \multicolumn{5}{|c|}{ Males HNR } \\
\hline & \multicolumn{5}{|c|}{ Mean \pm SD } \\
\hline & $<9$ & {$[9 ; 20]$} & {$[20 ; 26]$} & $\geq 26$ & $p$ \\
\hline$/ \mathrm{a} /$ & $0.15 \pm 0.01$ & $0.14 \pm 0.01$ & $0.14 \pm 0.02$ & $0.13 \pm 0.01$ & 0.272 \\
\hline /i/ & $0.10 \pm 0.08$ & $0.11 \pm 0.03$ & $0.11 \pm 0.02$ & $0.12 \pm 0.02$ & 0.840 \\
\hline $10 /$ & $0.14 \pm 0.00$ & $0.13 \pm 0.01$ & $0.14 \pm 0.01$ & $0.13 \pm 0.01$ & 0.648 \\
\hline \multirow[t]{3}{*}{$/ \mathrm{u} /$} & $0.13 \pm 0.02$ & $0.10 \pm 0.04$ & $0.14 \pm 0.02$ & $0.11 \pm 0.03$ & 0.680 \\
\hline & \multicolumn{5}{|c|}{ Females HNR } \\
\hline & \multicolumn{5}{|c|}{ Mean \pm SD } \\
\hline$/ \mathrm{a} /$ & $0.11 \pm 0.03$ & $0.12 \pm 0.03$ & $0.13 \pm 0.05$ & $0.12 \pm 0.03$ & 0.934 \\
\hline /i/ & $0.08 \pm 0.03$ & $0.12 \pm 0.02$ & $0.09 \pm 0.03$ & $0.10 \pm 0.03$ & 0.145 \\
\hline$/ 0 /$ & $0.13 \pm 0.01$ & $0.13 \pm 0.05$ & $0.12 \pm 0.01$ & $0.12 \pm 0.02$ & 0.901 \\
\hline$/ \mathrm{u} /$ & $0.15 \pm 0.12$ & $0.21 \pm 0.31$ & $0.16 \pm 0.02$ & $0.17 \pm 0.21$ & 0.972 \\
\hline
\end{tabular}

\begin{tabular}{|c|c|c|c|c|c|}
\hline \multirow[t]{3}{*}{ Vowels } & \multicolumn{5}{|c|}{ Males $\mathrm{F}_{\mathrm{ftr}}(\mathrm{Hz})$} \\
\hline & \multicolumn{5}{|c|}{ Mean \pm SD } \\
\hline & $<9$ & {$[9 ; 20]$} & {$[20 ; 26]$} & $\geq 26$ & $p$ \\
\hline$/ \mathrm{a} /$ & $4.10 \pm 0.35$ & $1.54 \pm 3.08$ & $2.16 \pm 0.15$ & $5.96 \pm 2.75$ & 0.155 \\
\hline /i/ & $5.46 \pm 0.36$ & $1.15 \pm 2.30$ & $1.51 \pm 2.13$ & $4.45 \pm 3.30$ & 0.203 \\
\hline $\mid 0 /$ & $3.59 \pm 2.00$ & $2.85 \pm 2.03$ & $2.77 \pm 0.31$ & $2.54 \pm 3.09$ & 0.965 \\
\hline \multirow[t]{3}{*}{$/ \mathrm{u} /$} & $0.00 \pm 0.00$ & $1.13 \pm 2.28$ & $3.41 \pm 0.37$ & $4.46 \pm 1.29$ & $0.035^{*}$ \\
\hline & \multicolumn{5}{|c|}{ Females $F_{\mathrm{ftr}}(\mathrm{Hz})$} \\
\hline & \multicolumn{5}{|c|}{ Mean \pm SD } \\
\hline$/ \mathrm{a} /$ & $2.20 \pm 3.11$ & $3.85 \pm 1.92$ & $3.69 \pm 0.41$ & $2.96 \pm 2.00$ & 0.604 \\
\hline /i/ & $2.57 \pm 3.63$ & $3.72 \pm 2.45$ & $3.21 \pm 0.68$ & $2.91 \pm 2.01$ & 0.833 \\
\hline $10 /$ & $0.00 \pm 0.00$ & $2.61 \pm 2.35$ & $1.80 \pm 1.61$ & $2.67 \pm 1.64$ & 0.349 \\
\hline$/ \mathrm{u} /$ & $1.94 \pm 2.74$ & $2.68 \pm 2.44$ & $2.78 \pm 4.81$ & $3.27 \pm 1.42$ & 0.900 \\
\hline
\end{tabular}

\begin{tabular}{|c|c|c|c|c|c|}
\hline & \multicolumn{5}{|c|}{ Males Mean \pm SD } \\
\hline & $<9$ & {$[9 ; 20]$} & {$[20 ; 26]$} & $\geq 26$ & $p$ \\
\hline MPD & $17.36 \pm 2.35$ & $25.21 \pm 6.57$ & $16.59 \pm 14.55$ & $18.20 \pm 7.01$ & 0.502 \\
\hline \multirow[t]{2}{*}{$s / z$} & $0.69 \pm 0.30$ & $1.02 \pm 0.42$ & $0.84 \pm 0.06$ & $0.78 \pm 0.43$ & 0.746 \\
\hline & \multicolumn{5}{|c|}{ Females Mean \pm SD } \\
\hline MPD & $13.70 \pm 3.63$ & $17.51 \pm 6.65$ & $12.52 \pm 3.63$ & $16.47 \pm 4.15$ & 0.496 \\
\hline$s / z$ & $1.00 \pm 0.51$ & $0.86 \pm 0.11$ & $0.91 \pm 0.38$ & $0.81 \pm 0.116$ & 0.635 \\
\hline
\end{tabular}

same pattern was observed for males but in shimmer measure only for $/ \mathrm{i} /(p<0.05)$ [Tables 6 and 7 ]. These perturbation measure findings might reflect sight differences of mass, tension and biomechanical characteristics of the vocal folds as a consequence of occupational ILFN-exposure. Although this could be related with the abnormal growth of collagen changes of the BMZ, confirmation would require histological analyses on further investigation.

For females, statistical analyses revealed a significant interaction between age and years of occupational ILFNexposure $(p<0.05)$ on perturbation measures (jitter and shimmer). The box plot analysis [Figures 1-3] revealed 


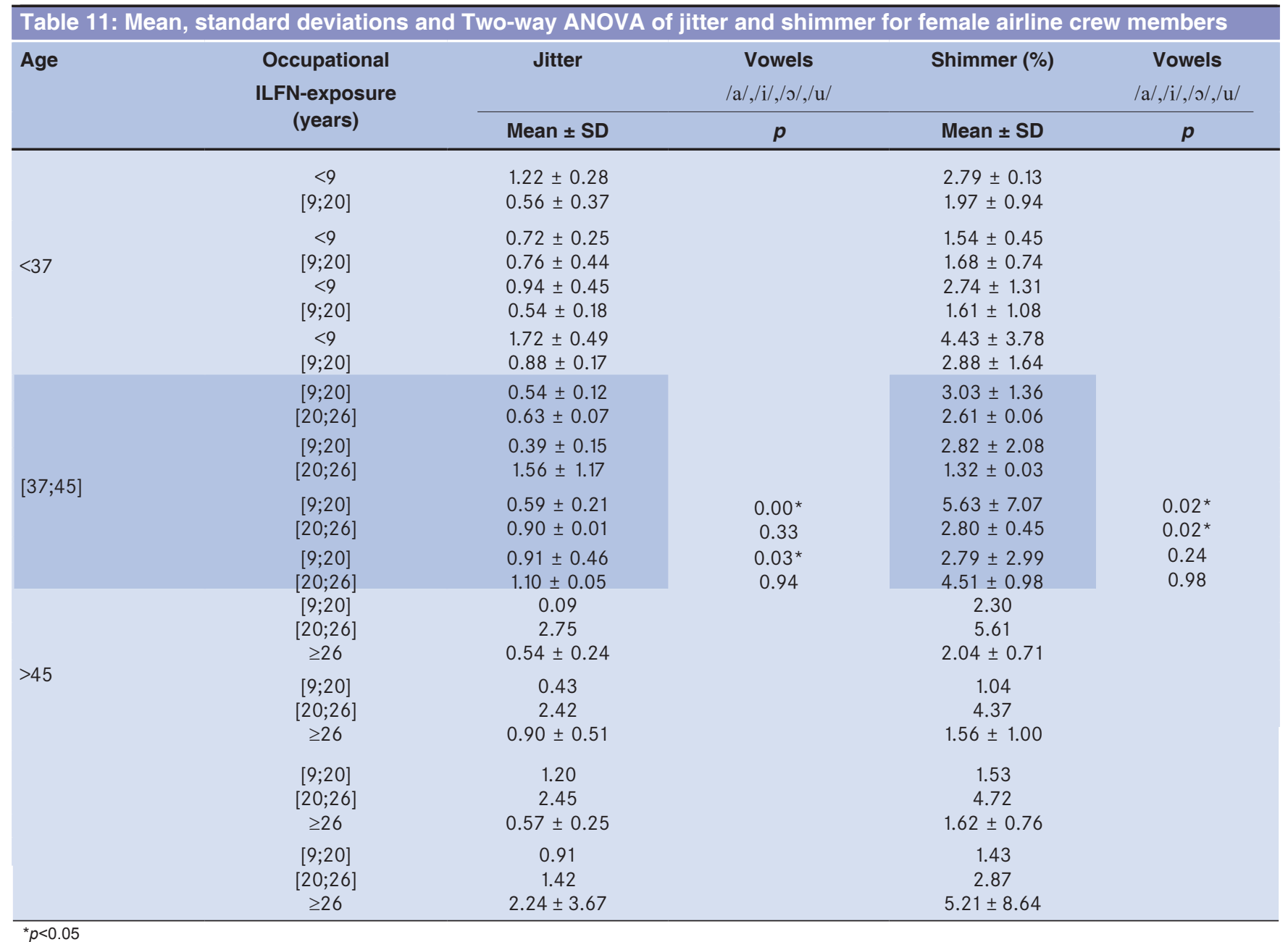

that jitter (\%) increased on the $[20 ; 26]$ occupational ILFNexposure interval and decreased on the $>26$ interval. The results may be indicative of a biological adaptation to ILFN environments, as it was suggested in others VAD studies that looked at the cardiac system. ${ }^{[3] \mid}$ This adaptation may consist on an unusual reorganization of the extra layers of tissue so that the voice acoustic parameters are no longer revealing significant differences. Another interpretation of these results may involve the concept of "survivorship bias". Individuals who are still on active duty after $>26$ years of occupational activity means that they have not been subjected to disability retirement or change of professional duties. This has been observed in other studies involving cabin crewmembers. ${ }^{[32]}$

The female subjects were smokers and this habit can affect voice perturbation measures. However, previous studies on VAD patients or older ILFN-exposure workers reported no differences between smokers and non-smokers in respiratory symptoms and signs. ${ }^{[2,15,16]}$
Nevertheless, when compared with the norms, the cabin crew members presented significant lower means $(p<0.05){ }^{\mid 21-23]}$ Temporal measures of MPD and S/Z ratio were steady throughout the four occupational intervals for both genders. That means that the possible inefficient coordination between the respiratory and phonatory systems and glottal closure inefficiency were not related to ILFN-exposure and/ or age.

Spectural measure of $\mathrm{F}_{\text {frt }}$, did not present a significant change throughout the four occupational and three age intervals revealing the integrity of neural controls for voice production.

This study under goes with other occupational ILFNexposure population studies (i.e., respiratory, cardiovascular, neurological). Cabin crew members presented acoustic patterns that might suggest the existence of small morphological and physiological changes of the respiratory and laryngeal systems. The laryngeal system could be, itself a target for ILFN acting silently on collagen and elastin also presented on vocal 


\begin{tabular}{|c|c|c|c|c|c|c|c|}
\hline \multirow[t]{2}{*}{ Age } & \multirow{2}{*}{$\begin{array}{l}\text { Occupational } \\
\text { ILFN-exposure } \\
\text { (years) }\end{array}$} & $F_{0}$ & $\begin{array}{c}\text { Vowels } \\
/ \mathrm{a} /, / \mathrm{i} /, / \mathrm{s} /, / \mathrm{u} /\end{array}$ & HNR & \multirow{2}{*}{$\begin{array}{c}\text { Vowels } \\
/ \mathrm{a} /, / \mathrm{i} /, / \mathrm{o} /, / \mathrm{u} / \\
\boldsymbol{p}\end{array}$} & $F_{\mathrm{ftr}}$ & \multirow{2}{*}{$\begin{array}{c}\begin{array}{c}\text { Vowels } \\
/ \mathrm{a} /, / \mathrm{i} /, / \mathrm{o} /, / \mathrm{u} /\end{array} \\
\boldsymbol{p}\end{array}$} \\
\hline & & Mean \pm SD & $p$ & Mean \pm SD & & Mean \pm SD & \\
\hline \multirow{4}{*}{$<37$} & $\begin{array}{c}<9 \\
{[9 ; 20]}\end{array}$ & $\begin{array}{r}207.40 \pm 10.63 \\
196.13 \pm 37.41\end{array}$ & & $\begin{array}{l}0.11 \pm 0.03 \\
0.12 \pm 0.03\end{array}$ & & $\begin{array}{l}2.20 \pm 3.11 \\
3.73 \pm 2.37\end{array}$ & \\
\hline & $\begin{array}{c}<9 \\
{[9 ; 20]}\end{array}$ & $\begin{array}{l}211.81 \pm 14.38 \\
201.23 \pm 36.00\end{array}$ & & $\begin{array}{l}0.08 \pm 0.03 \\
0.12 \pm 0.03\end{array}$ & & $\begin{array}{l}2.57 \pm 3.63 \\
2.79 \pm 2.53\end{array}$ & \\
\hline & $\begin{array}{c}<9 \\
{[9 ; 20]}\end{array}$ & $\begin{array}{c}202.97 \pm 9.52 \\
196.32 \pm 36.06\end{array}$ & & $\begin{array}{l}0.13 \pm 0.01 \\
0.12 \pm 0.03\end{array}$ & & $\begin{array}{l}0.00 \pm 0.00 \\
2.99 \pm 2.72\end{array}$ & \\
\hline & $\begin{array}{c}<9 \\
{[9 ; 20]}\end{array}$ & $\begin{array}{c}222.02 \pm 1.58 \\
207.97 \pm 35.75\end{array}$ & & $\begin{array}{l}0.15 \pm 0.12 \\
0.12 \pm 0.05\end{array}$ & & $\begin{array}{l}1.94 \pm 2.74 \\
2.65 \pm 2.34\end{array}$ & \\
\hline \multirow{4}{*}[37;45]{} & $\begin{array}{c}{[9 ; 20]} \\
{[20 ; 26]}\end{array}$ & $\begin{array}{r}183.18 \pm 39.05 \\
190.38 \pm 36.33\end{array}$ & & $\begin{array}{l}0.12 \pm 0.03 \\
0.11 \pm 0.05\end{array}$ & & $\begin{array}{l}4.16 \pm 1.65 \\
3.72 \pm 0.57\end{array}$ & \\
\hline & $\begin{array}{c}{[9 ; 20]} \\
{[20 ; 26]}\end{array}$ & $\begin{array}{l}201.48 \pm 20.28 \\
224.42 \pm 35.46\end{array}$ & & $\begin{array}{l}0.13 \pm 0.02 \\
0.08 \pm 0.04\end{array}$ & & $\begin{array}{l}5.50 \pm 1.59 \\
3.10 \pm 0.91\end{array}$ & \\
\hline & $\begin{array}{c}{[9 ; 20]} \\
{[20 ; 26]}\end{array}$ & $\begin{array}{c}181.98 \pm 29.97 \\
185.24 \pm 42.56\end{array}$ & $\begin{array}{l}0.89 \\
0.58\end{array}$ & $\begin{array}{l}0.15 \pm 0.07 \\
0.13 \pm 0.01\end{array}$ & $\begin{array}{l}0.06 \\
0.24\end{array}$ & $\begin{array}{l}1.64 \pm 1.93 \\
1.55 \pm 2.19\end{array}$ & $\begin{array}{l}0.84 \\
0.32\end{array}$ \\
\hline & $\begin{array}{c}{[9 ; 20]} \\
{[20 ; 26]}\end{array}$ & $\begin{array}{r}191.09 \pm 26.58 \\
221.99 \pm 40.32\end{array}$ & $\begin{array}{l}0.88 \\
0.84\end{array}$ & $\begin{array}{l}0.38 \pm 0.50 \\
0.17 \pm 0.03\end{array}$ & $\begin{array}{l}0.62 \\
0.57\end{array}$ & $\begin{array}{l}1.92 \pm 2.56 \\
0.00 \pm 0.00\end{array}$ & $\begin{array}{l}0.60 \\
0.20\end{array}$ \\
\hline \multirow{4}{*}{$>45$} & $\begin{array}{c}{[9 ; 20]} \\
{[20 ; 26]} \\
\geq 26\end{array}$ & $\begin{array}{c}234.66 \\
229.34 \\
213.90 \pm 67.98\end{array}$ & & $\begin{array}{c}0.80 \\
0.17 \\
0.12 \pm 0.03\end{array}$ & & $\begin{array}{c}3.36 \\
3.64 \\
2.96 \pm 2.00\end{array}$ & \\
\hline & $\begin{array}{c}{[9 ; 20]} \\
{[20 ; 26]} \\
\geq 26\end{array}$ & $\begin{array}{c}248.84 \\
221.75 \\
229.56 \pm 72.65\end{array}$ & & $\begin{array}{c}0.10 \\
0.11 \\
0.10 \pm 0.03\end{array}$ & & $\begin{array}{c}2.17 \\
3.45 \\
2.91 \pm 2.01\end{array}$ & \\
\hline & $\begin{array}{c}{[9 ; 20]} \\
{[20 ; 26]} \\
\geq 26\end{array}$ & $\begin{array}{c}219.18 \\
234.48 \\
208.10 \pm 61.13\end{array}$ & & $\begin{array}{c}0.11 \\
0.12 \\
0.12 \pm 0.02\end{array}$ & & $\begin{array}{c}4.21 \\
2.30 \\
2.67 \pm 1.64\end{array}$ & \\
\hline & $\begin{array}{c}{[9 ; 20]} \\
{[20 ; 26]} \\
\geq 26\end{array}$ & $\begin{array}{c}225.95 \\
238.60 \\
222.67 \pm 72.12\end{array}$ & & $\begin{array}{c}0.12 \\
0.15 \\
0.17 \pm 0.21\end{array}$ & & $\begin{array}{c}5.88 \\
8.33 \\
3.27 \pm 1.42\end{array}$ & \\
\hline
\end{tabular}

\begin{tabular}{|c|c|c|c|c|c|}
\hline \multirow[t]{2}{*}{ Age } & \multirow{2}{*}{$\begin{array}{c}\text { Occupational } \\
\text { ILFN-exposure (years) }\end{array}$} & \multicolumn{2}{|c|}{ MPD } & \multicolumn{2}{|c|}{$S / Z$} \\
\hline & & Mean \pm SD & $p$ & Mean \pm SD & $p$ \\
\hline$<37$ & $\begin{array}{c}<9 \\
{[9 ; 20]}\end{array}$ & $\begin{array}{l}13.70 \pm 3.63 \\
16.20 \pm 5.52\end{array}$ & & $\begin{array}{l}1.00 \pm 0.51 \\
0.87 \pm 0.13\end{array}$ & \\
\hline$[37 ; 45]$ & $\begin{array}{c}{[9 ; 20]} \\
{[20 ; 26]}\end{array}$ & $\begin{array}{l}18.94 \pm 7.22 \\
14.39 \pm 2.34\end{array}$ & 0.24 & $\begin{array}{l}0.83 \pm 0.12 \\
0.74 \pm 0.30\end{array}$ & 0.19 \\
\hline$>45$ & $\begin{array}{c}{[9 ; 20]} \\
{[20 ; 26]} \\
\geq 26\end{array}$ & $\begin{array}{c}22.08 \\
8.78 \\
16.47 \pm 4.15\end{array}$ & & $\begin{array}{c}0.93 \\
1.27 \\
0.81 \pm 0.16\end{array}$ & \\
\hline
\end{tabular}

folds. Previous bronchoscopic studies revealed pink patches and blood ramifications without bleeding of the trachea and vocal folds as well as bleeding events limited to the lower portion of the epithelium..$^{[11,12]}$ Subsequent histological studies performed on biopsies of these pink patches and non-pink patches (as seen through bronchoscopy) revealed abnormal cellular architectures, as well as the characteristic proliferation of collagen and elastin fibers in the absence of an inflammatory process. ${ }^{[33]}$ The results of the study herein further lend evidence to the importance of mechanotransduction cellular signaling in ILFN-induced pathology. ${ }^{[34]}$
VAD patients present non-productive cough, hoarseness, repeated upper and lower respiratory infections, bronchitis and respiratory insufficiency. ${ }^{[2,11,16]}$ These symptoms and signs have an effect on their voices. Cabin crewmembers are considered Type II-Professional voice, meaning that voice is an integral part of their job. ${ }^{[24]}$ Voice can be assessed using acoustic analysis which is a non-invasive, low cost and sensitive method to detect changes in the laryngeal and respiratory systems. Therefore, voice acoustic analyses is a highly appropriate tool to track down "silent" voice symptoms associated with ILFN-exposure. 
The present study revealed voice acoustic patterns related to occupational ILFN-exposure and age, some of them significant. Future longitudinal studies are needed crossing voice acoustic data with histological vocal fold data to confirm the hypothesis raised about the effect of ILFNexposure and the production of extra-cellular matrix collagen within the laryngeal system. Voice acoustic analysis may be an important tool for confirming a VAD diagnosis.

\section{CONCLUSIONS}

VAD is characterized by abnormal growth of extra-cellular matrix collagen and elastin in the absence of an inflammatory process, and is caused by long-term ILFN- exposure (specifically frequencies below $500 \mathrm{~Hz}$, including infrasound). VAD can develop due to occupational exposure to ILFN, such as with aircraft pilots, crewmembers, and technicians; ship machinists, restaurant workers, and DJ's.

In the present study, cabin crewmembers' perturbation measures presented significant difference among the four professional activity years $(p<0.05)$ for both genders. Additionally, females presented an interaction between age and occupational ILFN-exposure $(p<0.05)$. This pattern may be indicative of histological changes within the laryngeal system, specifically on the vocal folds, and related to long term ILFN-exposure.

As future research, longitudinal studies are recommended on voice acoustic analyses across the professional activity years on the same subjects to verify how these parameters change over the ILFN-exposure years, and whether this type of evaluation could be included as a complementary diagnostic tool for VAD.

\section{ACKNOWLEDGMENTS}

This work was supported, in part, by the Institute of Electronics and Telematics Engineering of Aveiro. The authors wish to thank to Marlene Mocho, António Galvão and Oliveira Carvalho for the recordings, to Carolina Santos for the acoustic analysis, to Prof. António Sardinha for his assistance in statistic analysis and to Sindicato Nacional do Pessoal de Voo da Aviação Civil for providing the space in which the recordings were performed. The authors also thank all cabin crew members who voluntarily participated in this study.

\section{REFERENCES}

1. Branco NA, Alves-Pereira M. Vibroacoustic disease. Noise Health 2004;6:3-20.

2. Castelo Branco NA. The clinical stages of vibroacoustic disease. Aviat Space Environ Med 1999;70:32-9.

3. Shabetai R, Diseases of the pericardium. In: Hurst's The Heart, Arteries and Veins. Schlant RC, Wayne Alexander R, (eds) editors, McGraw-Hill, New York; 1994, p. 1547-1649, 1994.
4. Marciniak W, Rodriguez E, Olszowska K, Atkov O, Botvin I, Araujo A, et al. Echocardiography evaluation in 485 aeronautical workers exposed to different noise environments. Aviat Space Environ Med 1999;70:46-53.

5. Martinho Pimenta, A, Castelo Branco, NAA,. Neurological aspects of vibroacoustic disease, Aviat. Space Environ. Med., 1999;70 (March, Suppl),: A91-95, 1999.

6. Pimenta MG, Martinho Pimenta AJ, Castelo Branco MS, Castelo Branco NA. ERP P300 and brain magnetic resonance imaging in patients with vibroacoustic disease. Aviat Space Environ Med 1999;70 Suppl 3:A107-14.

7. Fonseca J, Martins dos Santos J, Oliveira P, Laranjeira N, Águas A, Castelo Branco NA. Noise-induced gastric lesions: A light and electron microscopy study of the rat gastric wall exposed to low frequency noise. Arq Gastroenterol 2012;49:82-8.

8. Silva MJ, Carothers A, Castelo Branco NA, Dias A, Boavida MG. Sister chromatid exchange analysis in workers exposed to noise and vibration. Aviat Space Environ Med 1999;70:40-5.

9. Águas AP, Esaguy N, Grande N, Castro AP, Castelo Branco NA. Effect low frequency noise exposure on BALB/c mice splenic lymphocytes. Aviat Space Environ Med 1999;70:128-31.

10. Alves-Pereira M, Reis Ferreira JM, Joanaz de Melo J, Motylewski J, Kotlicka E, Castelo Branco NA. Noise and the respiratory system. Rev Port Pneumol 2003;9:367-79.

11. Branco NA, Ferreira JR, Alves-Pereira M. Respiratory pathology in vibroacoustic disease: 25 years of research. Rev Port Pneumol 2007;13:129-35.

12. Monteiro M, Reis Ferreira J, Mendes CP, Alves-Pereira M, Castelo Branco NAA. Vibroacoustic disease and respiratory pathology III - Tracheal \& bronchial lesions. Proceedings Internoise 2004. Prague, Czech Republic, 2004; No. 638, 5 pages. (ISBN: 80-0103055-5)

13. Castelo Branco NA. A unique case of vibroacoustic disease: A tribute to an extraordinary patient. Aviat Space Environ Med 1999;70:27-31.

14. Castelo Branco NAA, Alves-Pereira M, Martins dos Santos J, Monteiro E. SEM and TEM study of rat respiratory epithelia exposed to low frequency noise. In: Science and Technology Education in Microscopy: An Overview, A. Mendez-Vilas, (Ed.) editor, Formatex: Badajoz, Spain, 2003; Vol. II: 505-33.

15. Reis Ferreira J, Mendes CP, Castelo Branco NA, Monteiro E, Alves Pereira M. The human lung and pleura in vibroacoustic disease. Proc $8^{\text {th }}$ Intern Conf Noise as a Public Health Problem (ICBEN) Rotterdam, Holland; 2003. p. 386-7. (ISBN: 90-807990-1-7)

16. Reis Ferreira JM, Couto AR, Jalles-Tavares N, Castelo Branco MS, Castelo Branco NA. Airflow limitations in patients with vibroacoustic disease. Aviat Space Environ Med 1999;70:63-9.

17. Hirano M. Morphological structure of the vocal cord as a vibrator and its variations. Folia Phoniatr (Basel) 1974;26:89-94.

18. Gray S, Hirano M, Satto K. Molecular and cellular struture of the vocal fold tissue. In I.R. Titze editor. Vocal fold physiology: Frontiers in basic science: San Diego: Singular Publishing Group;1993.p. 1-36.

19. Mendes A, Castro E. Análise Acústica da Avaliação Vocal I: Tarefas fonatórias e medidas acústicas. Rev Port Otorrinolaringol Cirurgia Cérvico Facial 2005;43:127-36.

20. Colton R, Casper J. Understanding voice problems: A physiological perspective for diagnosis and treatment. Baltimore: Williams and Wilkins; 1990.

21. Mendes AP, Alves Pereira M, Castelo Branco NA. Voice acoustic patterns of patients diagnosed with vibroacoustic disease. Rev Port Pneum 2006;12:375-82.

22. Mendes AP, Santos $\mathrm{CP}$, Graça A, Galvão A, Carvalho RO, Sousa MJ, et al. Voice acoustic analyses of commercial airline pilots. 
Proc Internoise, Shanghai, China; 2008; No. IN-08-591. 11 pages. (ISBN 9781605609898)

23. Mendes AP, Graça A, Santos CP, Galvão A, Carvalho RO, Sousa MJ, Alves Pereira M, Castelo Branco N. Voice acoustic analyses in airline cabin crewmembers. Proc Internoise, Shanghai, China:2008; No. IN-08-595., 13 pages. (ISBN 9781605609898)

24. Koufman JA, Isacson G. Voice disorders. Philadelphia: WB Saunders; 1991.

25. Mendes AP, Costa A, Martins A, Fernandes A, Vicente S, Freitas T. Contributo para a construção de um Texto Foneticamente Equilibrado para o Português Europeu. Revista CEFAC Actualização Científica em Fonoaudiologia e Educação p. 98-11. In press.

26. Scherer RC, Vail VJ, Guo CG. Required number of tokens to determine representative voice perturbation values. J Speech Hear Res 1995;38:1260-9.

27. Titze IR, Winholtz WS. Effect of microphone type and placement on voice perturbation measurements. J Speech Hear Res 1993; 36:1177-90.

28. CSL Kay, PENTAX. Computerized Speech Lab (Version 4500) [Computer Software]. Lincoln Park, NJ: A Division of Pentax Medical Company, 2007.

29. Titze I. Principles of the voice production. Englewood Cliffs, NJ: Prentice Hall, 1994.

30. Ventry I, Schiavetti N. Evaluating research in speech pathology and audiology. $2^{\text {nd }}$ ed New York: Macmillan; 1986

31. Alves-Pereira M, Joanaz de Melo J, Castelo Branco, NAA. Pericardial biomechanical adaptation to low frequency noise stress. IN: A. Méndez-Vilas (ed.) Recent Advances in Multidisciplicnary Applied Physics. Elsevier: London, 2005 p. 363-7.

32. Araujo A, Pais F, Lopo Tuna JMC, Alves-Pereira M, Castelo Branco NAA. Echocardiography in noise-exposed flight crew. Proc Internoise 2001, The Hague, Holland, 2001:1007-10.

33. (ISBN: 9080655422)33. Reis Ferreira J, Monteiro MB, Tavares F, Serrano I, Monteiro E, Mendes CP, Alves-Pereira M, Castelo Branco $N A A$ et al. Involvement of central airways in vibroacoustic disease - Thomé Villar/Boehringer Ingelheim Award 2004. Rev Port Pneu 2006;12(2):93-105.

34. Alves-Pereira M, Castelo Branco NAA. Vibroacoustic disease: Biological effects of infrasound and low frequency noise explained by mechanotransduction cellular signaling. Prog Biophy Molec Biol 2007;93:256-79.

Cite this article as: Mendes A, Graça A, Jorge A, Alves-Pereira M, Castelo Branco NA, Freitas A, et al. The effects of ILFN-exposure on voice acoustic parameters of commercial cabin crewmembers. J Laryngol Voice 2012;2:70-80.

Source of Support: This work was supported, in part, by the Institute of Electronics and Telematics Engineering of Aveiro. Conflict Interest: No.

\section{Author Help: Online submission of the manuscripts}

Articles can be submitted online from http://www.journalonweb.com. For online submission, the articles should be prepared in two files (first page file and article file). Images should be submitted separately.

1) First Page File:

Prepare the title page, covering letter, acknowledgement etc. using a word processor program. All information related to your identity should be included here. Use text/rtt/doc/pdf files. Do not zip the files.

2) Article File:

The main text of the article, beginning with the Abstract to References (including tables) should be in this file. Do not include any information (such as acknowledgement, your names in page headers etc.) in this file. Use text/rtf/doc/pdf files. Do not zip the files. Limit the file size to $1024 \mathrm{~kb}$. Do not incorporate images in the file. If file size is large, graphs can be submitted separately as images, without their being incorporated in the article file. This will reduce the size of the file.

3) Images:

Submit good quality color images. Each image should be less than $\mathbf{4 0 9 6} \mathbf{~ k b}$ (4 MB) in size. The size of the image can be reduced by decreasing the actual height and width of the images (keep up to about 6 inches and up to about $1800 \times 1200$ pixels). JPEG is the most suitable file format. The image quality should be good enough to judge the scientific value of the image. For the purpose of printing, always retain a good quality, high resolution image. This high resolution image should be sent to the editorial office at the time of sending a revised article.

4) Legends:

Legends for the figures/images should be included at the end of the article file. 